\title{
ENFERMAGEM OBSTÉTRICA NO ACOMPANHAMENTO PRÉ - NATAL
}

\author{
THE OBSTETRIC NURSING IN THE PRENATAL CONSULTATION \\ LAENFERMERÍA OBSTÉTRICAEN ELACOMPAÑAMIENTO PRENATAL
}

\author{
Lucia Helena Garcia Penna ${ }^{1}$ \\ Jane Márcia Progianti \\ Luiza Mara Correa ${ }^{3}$
}

RESUMO: Consiste em um relato de experiência acerca de um projeto de pesquisa iniciado em agosto de 1997, envolvendo docentes do Departamento Materno-infantil da Faculdade de Enfermagem da UERJ e profissionais do Centro Municipal de Saúde do Rio de Janeiro da S.M.S./RJ. Tem por objetivo, descrever o processo de inserção da Consulta de Enfermagem de Pré-natal no conteúdo da graduação, bem como sua estruturação e implantação no referido Centro Municipal de Saúde. Esse trabalho propõe rever também, o modelo biomédico das consultas, a fim de proporcionar reflexões e rupturas de paradigmas, e com isso auxiliar no aprendizado e na ampliação do acompanhamento de pré-natal no Rio de Janeiro

PALAVRAS CHAVE: consulta de enfermagem, pré-natal, enfermagem, obstétrica

\section{INTRODUÇÃO}

Tem sido amplamente divulgado a situação em que o Brasil encontra-se no que diz respeito à saúde da mulher, particularmente em seu período reprodutivo.

A OMS divulgou por ocasião do Dia Mundial da Saúde (celebrado a 7 de abril de 1998), cujo tema desse ano foi "Maternidade Segura", um relatório diagnóstico afirmando que, "nos paises onde a mortalidade matema permanece alta, as mulheres sofrem principalmente porque não têm acesso a cuidados médicos básicos durante a gravidez, no momento do nascimento do bebê e no período pós-parto". O levantamento mostra que,"nos chamados países em desenvolvimento, como o Brasil, apenas $53 \%$ dos partos ocorrem na presença de um profissional qualificado, seja, médico, enfermeiro ou parteira legalmente credenciada". (FIOCRUZIMS, 1998, p. 2 ).

Segundo Fontes (1984, p.12), o Pré-natal visa o bem estar global do binômio mãe-filho, na fase mais crucial da existência humana. Para isso a ciência médica desenvolveu técnicas, procedimentos, condutas permissivas de detecção e correção dos diversos distúrbios que, incidindo sobre um deles ou ambos, possam impedir, dificultar, bloquear o curso normal da gravidez. O Pré-natal tem como objetivo básico reduzir a morbimortalidade materna e fetal.

A assistência Pré-natal constitui o rol de cuidados, condutas e procedimentos em favor da mulher grávida e do concepto, durante o período acima mencionado. Na prática, entretanto, o termo pré-natal consagrou-se como, sinteticamente, a orientação e assistência gestacional.

\footnotetext{
1 Profa do Departamento de Enfermagem Materno-Infantil da Faculdade de Enfermagem da UERJ - Mestre em Enfermagem

2 Prof $^{a}$ do Departamento de Enfermagem Materno-Infantil da Faculdade de Enfermagem da UERJ - Mestre em Enfermagem

${ }^{3}$ Profa do Departamento de Enf. Materno-Infantil da Faculdade de Enfermagem da UERJ
} 
O Ministério da Saúde, destacado em Nogueira (1994, p.17) afirma que "apenas $19 \%$ dos estados brasileiros apresentam cobertura de pré-natal acima de $40 \%$; somente $53 \%$ das grávidas têm um acompanhamento pré-natal de quatro consultas durante a gestação; $9,7 \%$ das mortes maternas no Brasil resultam de complicações decorrentes do aborto provocado; $44,1 \%$ dos partos realizados nos hospitais públicos e conveniados do Inamps foram cesarianas e grande parte delas realizada por meio de indicação abusiva".

Considerando ser a área materno-infantil prioritária nos programas de saúde, constituindo $70,98 \%$ da população brasileira, faz-se necessário a atuação efetiva da equipe de enfermagem. Ziegele Cranley(1985, p. 253) afirmam que, "a gestante apresenta no transcurso da gravidez, uma série de necessidades que o enfermeiro precisa estar apto a preencher". Com isso ressaltase a importância do enfermeiro junto a outros profissionais; na tentativa de melhorar o nivel assistencial às mulheres no ciclo grávido-puerperal, colaborando assim para minimizar as intercorrências obstétricas; assegurando o bem-estar físico e mental da cliente e do concepto.

A partir desta panorâmica e entendendo que a prática da Enfermagem Obstétrica possui dentre outros, o compromisso de modificar-se e inovar-se de acordo com as mudanças sociais e políticas que vem sofrendo a população feminina, principalmente frente às suas conquistas e reivindicações, acreditamos que a realização da Consulta de Enfermagem no Atendimento Prénatal, constitui um dos grandes recursos para a contribuição da expansão da cobertura assistencial às gestantes. Entretanto é importante enfatizar, que esta deve estar calcada nos principios da Educação em Saúde, participativa, valorizando a integralidade da assistência à mulher, a fim de favorecer sua qualidade de vida.

Defendemos que na formação do profissional enfermeiro(a), este desenvolva suas atividades baseadas em práticas educativas, que não exclua o modelo tradicional (modelo biomédico), mas supere-o, associando a ele práticas assistenciais/educativas que valorizem realmente o saber popular, as reais necessidades da(o) cliente, particularmente a mulher, a fim de que sejam assistidas de forma integral, valorizando seu corpo e não somente examinandoo e docilizando-o, relativizando assim o poder do profissional através do saber (poder do saber/ institucional).

Desta forma objetivamos descrever sobre a experiência da inserção da Consulta de Enfermagem no Curso de Graduação em Enfermagem da UERJ, no conteúdo programático da graduação, na disciplina de Enfermagem Obstétrica e Saúde da Mulher, uma estratégia de ensino inovadora, visando despertar nos futuros profissionais o papel educativo/participativo, valorizando a clientela como cidadã, responsável pelo seu corpo e sua saúde.

\section{REFERENCIAL TEÓRICO}

Ao desenvolvermos este estudo, procuramos nos subsidiar de alguns conceitos e reflexões sobre o Programa de Assistência Integral à Saúde da Mulher (PAISM), sobre Assistência Prénatal, Educação em Saúde e Consulta de Enfermagem.

O processo de democratização no qual passou o País no início dos anos 80 , refletiu em vários setores da sociedade. No âmbito da saúde, através da influência de profissionais feministas no Ministério da Saúde e do próprio movimento social de mulheres, algumas conquistas começaram a ser desenhadas. Uma delas foi a elaboração do Programa de Assistência Integral à Saúde da Mulher (BRASIL,1984, p. 12) que possui como bases de ações programáticas a assistência ao pré-natal, parto e puerpério, prevenção e controle do câncer cérvico-uterino e de mama e planejamento familiar.

A elaboração do Programa de Assistência Integral à Saúde da Mulher (PAISM) "rompe com o modelo denominado materno-infantil, que focaliza a mulher como cliente especial, em função do seu papel na reprodução biológica..., o PAISM também propõe, novas formas de relacionamento entre os profissionais de saúde e as usuárias (...) devendo, estar baseado no 
respeito mútuo". (Ávila, 1995, p. 7)

De acordo com o PAISM/MS (BRASIL,1984, p. 13), a assistência pré-natal "constitui um conjunto de procedimentos clínicos e educativos com o objetivo de promover a saúde e identificar precocemente os problemas que possam resultar em risco para a saúde da gestante e do concepto.

Sabemos que as ações preconizadas pelo Ministério da Saúde, necessitam para serem implementadas, da adoção de estratégias que assegurem o aumento da cobertura e a melhoria da qualidade assistencial.

Faleiros (1993, p. 28) destaca que "saber utilizar os recursos institucionais em função dos interesses da população vem se tornando um desafio cada vez maior da atuação profissional. Exige-se hoje do profissional não mais uma competência individual, que o marcou outrora como o bom profissional, mas uma reflexão coletiva para saber o momento oportuno de avançar e de recuar na sua estratégia institucional e em relação aos grupos populares".

No setor saúde, distinguimos duas realidades distintas: de um lado, o profissional de saúde, detentor do "saber", com um relativo nível sócio-econômico; do outro, a clientela, "ignorante", na maioria com um nível sócio-econômico desfavorável, ou seja, um profissional com a função de oferecer informações a um grupo, quando, na maioria das vezes, ele desconhece as verdadeiras necessidades dessa população.

A partir desta distinção, muitas são as conseqüências na interelação pessoal de ajuda. Observamos, às vezes, que esse profissional de saúde por motivo de defesa, e de dominação, prefere anular os conhecimentos e necessidades dessa população e oferecer um "pacote" assistencial pronto, o qual não permite haver avaliações e críticas quanto ao seu conhecimento.

É importante enquanto profissional da saúde refletir e buscar novas maneiras de se promover melhor qualidade de vida à clientela, entretanto deve-se estar atento quanto aos reais interesses ideológicos da realização de uma simples atividade.

A dimensão educativa é, sem dúvida, um dos aspectos mais inovadores do PAISM, pois objetiva contribuir com o acréscimo de informações que as mulheres possuem sobre seu corpo, e valoriza suas experiências de vida. As práticas educativas buscam recuperar a mulher como ser integral, como sujeito de sua saúde e de sua própria vida.

Penna (1996, p. 38) afirma que as práticas educativas, consideradas o eixo condutor de todas as ações programáticas do PAISM, possuem como objetivo maior, o de capacitar a mulher no conhecimento do seu corpo e no estímulo ao auto cuidado, abordando às questões da sexualidade, do corpo, do gênero, trazidas do cotidiano dessa mulher. O PAISM preconiza que as ações educativas devam ser realizadas através de metodologias participativas; pois, "mais importante que oferecer conteúdos objetivos (informações sobre sexualidade, maternidade, menstruação, aborto e etc.) é precisar uma forma de relação com a mulher e com o corpo dela, e isto implica num método de trabalho diferente do usual nos serviços de saúde" (BRASIL,1993, p. 15).

O ensino da Enfermagem Obstétrica assim como sua prática vem sofrendo transformações e inovações em virtude dos acontecimentos e movimentos histórico-sociais, particularmente, os que dizem respeito à população feminina. Tal fato, vem exigindo estudos críticos de seus principios básicos, do valor de suas práticas anteriores e até mesmo das atuais, afim de oferecer uma visão mais crítica, propondo novos métodos e procedimentos para um melhor atendimento às necessidades de uma mulher.

Com isso, entendemos a Enfermagem Obstétrica, com uma nova visão social, objetivando a promoção da saúde da mulher, não somente valorizando o enfoque reprodutivista, biológico e sim , O TODO, considerando sempre o contexto sócio-político-econômico e cultural onde está inserida.

No que diz respeito à assistência ao pré-natal, é indiscutível a participação da(o) enfermeira(o) na melhoria dos índices de qualidade de assistência às gestantes, bem como no 
aumento da cobertura assistencial do pré-natal. Contudo, sabemos que, geralmente, a maioria das Consultas de Enfermagem na Assistência ao Pré-natal seguem o modelo biomédico, ou seja, depois de obter o relato das queixas do cliente, investiga por meio de perguntas e exames todos os outros órgãos do corpo e o seu passado de vida; o profissional supõe alguns diagnósticos mais prováveis e pede exames de laboratório para chegar a um diagnóstico único; depois disso, traça o plano de tratamento e orienta o paciente.

Vasconcellos (1989, p. 26) nos aponta que esse modelo de consulta é criado mais para atender consultórios particulares por ser muito demorado e, que apesar de ser justificado como uma tentativa de conhecer de forma global o cliente, ele na verdade permite conhecer os órgãos do cliente. Dessa forma, analisamos que seria muito mais lucrativo, se nos voltássemos para uma relação de aprendizado e de aprofundamento sobre o significado da doença na vida do cliente.

Acreditamos que muitas das atividades desenvolvidas no modelo tradicional têm muito a contribuir, entretanto destacamos que é importante inovar à medida que novas conquistas, análises reflexivas e desenvolvimentos socio-histórico-políticos vão acontecendo.

Maranhão (1990:, p. 10) ressalta que as funções e atividades da Enfermeira Obstetra no ciclo gravídico-puerperal foram preconizadas desde 1973, pela Organização Pan-americana de Saúde/OMS, abrangendo as áreas assistenciais, educativas, administrativas e de pesquisa. Nessa ocasião, Cranch (1972, p. 34), afirma que "os serviços de enfermagem/obstetrícia contribuem consideravelmente para a assistência materno-infantil".

Não nos deteremos, nesta ocasião, a listar todas as atividades a serem desenvolvidas na Consulta de Enfermagem no Pré-Natal. Neste momento, objetivamos a priori registrar que a Consulta de Enfermagem no Pré-natal será desenvolvida em todas as suas fases, preconizadas pelo PAISM/MS e pela OPAS/OMS, valorizando uma nova estratégia didático-pedagógica e de assistência no pré-natal.

\section{ASPECTOS METODOLÓGICOS}

Este trabalho consiste em um relato de uma proposta didático-pedagógica e assistencial, realizada num Centro Municipal de Saúde do Rio de Janeiro, sendo desenvolvida por algumas professoras do Departamento de Enfermagem Materno-infantil da Faculdade de Enfermagem da UERJ, as quais são responsáveis por atividades das disciplinas de Enfermagem Obstétrica do Curso de Graduação.

As autoras ao refletirem sobre o ensino da enfermagem obstétrica, perceberam dentre outros aspectos, dificuldades em oferecer no conteúdo programático da disciplina de enfermagem obstétrica, do curso de graduação, a atividade didático-pedagógoca e assistencial da consulta de enfermagem no pré-natal. Algumas dessas dificuldades são justificadas pelo número de professores às vezes insuficiente para todos os campos (relação professor/aluno), pela disponibilidade de campos com perfil e estrutura didático-pedagógica, e consequentemente a isso, a valorização dessa atividade para os cursos de habilitação e especialização na área obstétrica.

Dessa forma, preocupadas em ampliar o conhecimento dos profissionais ainda no curso de graduação, objetivando sensibilizá-los quanto às necessidades da população feminina em idade fértil e, visando estabelecer novas práticas assistenciais/educativas na melhoria da qualidade de vida da clientela, resolveram iniciar em agosto de 1997, um Projeto de Consulta de Enfermagem no Pré-natal de Baixo Risco, junto à um Centro Municipal, onde desenvolveremos atividades de ensino, pesquisa e assistência. A inovação de nossa proposta consiste em associar o modelo biomédico da consulta de pré-natal com atividades inovadoras - práticas educativas, preconizadas basicamente pelo PAISM/MS.

A partir do início em agosto de 1997, atendemos cerca de 1000 gestantes, na faixa etária 
de 15 a 40 anos, tendo participado as professoras responsáveis e turmas do $6^{\circ}$ período (intemato) e $8^{\circ}$ periodo.

Atendemos as clientes, no momento, em apenas dois dias da semana, no turno da tarde. As mesmas são agendadas para a consulta de enfermagem atendendo aos critérios únicos do pré-natal de baixo risco do Centro Municipal. Desenvolvemos consultas de primeira vez e subseqüentes, visando a integralidade da cliente e, participando do trabalho da equipe multidisciplinar (odontologia, psicologia, assistente social, médico, nutricionista, farmacêutico, equipe de enfermagem, imunização, dermatologia). As pacientes que por ventura apresentarem algum risco clínico/obstétrico, são referenciadas por nós a outra unidade, ou seja, a uma matemidade de referência, visto que não corresponderiam mais ao nivel de assistência primária.

Nosso intuito não é, somente, de aumentar a quantidade do atendimento pré-natal, mas de oferecer uma nova proposta de atuaçăo, valorizando a qualidade e as práticas educativas; registrando sempre a desenvoltura de uma prática de ensino, onde visamos multiplicar uma qualidade de assistência através dos futuros profissionais de saúde.

Nossa intenção consiste em desenvolver no Pré-natal, a Consulta de Enfermagem em duas modalidades. Primeiramente, teremos a consulta individualizada, onde a gestante é admitida, sendo colhido sua anamnese, solicitado os exames preliminares da rotina, realizado o exame físico e obstétrico e analisado a necessidade de encaminhamentos, orientações e prescriçōes. Posteriormente, de acordo com sua idade gestacional, essa cliente deverá ser agendada para a consulta coletiva, realizada intercaladamente às consultas individualizadas, onde a gestante, respeitando sua idade gestacional, participará com outras clientes, de uma consulta onde valoriza-se alguns dos seus aspectos individuais, mas que tem por objetivo maior, oferecer às gestantes, o conhecimento sobre a gestação, sobre as modificações como o seu corpo e do filho que está sendo gerado. Nessa consulta, será abordado além de dinâmicas sobre o autoconhecimento, conhecimento sobre o seu corpo, sexualidade, gênero, realizaremos juntamente como as clientes, o exame obstétrico sumário, orientando, encaminhando e prescrevendo de acordo com as necessidades surgidas.

No momento, nos limitamos a realização da consulta de enfermagem no pré-natal, individualizada, como anteriormente mencionada. Faz parte do projeto, desenvolvermos Grupos de Gestantes, Grupo de Gestante Adolescentes, Grupo de Sala de Espera, Consultas de Enfermagem Coletivas no Pré-natal.

\section{CONSIDERAÇÕES FINAIS}

O presente trabalho encontra-se em andamento, mas já percebemos que o mesmo tem seu valor, ao oferecer reflexões sobre as atuais atividades da enfermagem obstétrica, tanto na área de ensino, de assistência, quanto na de pesquisa no pré-natal. Destaca-se pelas inovaçöes e perspectivas de rupturas de paradigmas, sempre objetivando uma melhoria da qualidade de vida da população feminina, objeto de nossa prática social.

ABSTRACT: This study consists of a report about a research project which started in August, 1997 The project involved teachers from the maternal- paediatrics department with Faculdade de Enfermagem - UERJ ( Nursing Faculty with State University of Rio de Janeiro) and professionals from the municipal health center in Rio de Janeiro - SMS/RJ. The introduction of prenatal nursing consultation as a new subject in the nursing graduation course, as well as its formation, and implementation in the referred municipal health center is reported in this paper. This study also proposes a review of the current biomedical consultation model, in order to motivate reflection, break paradigms, and increase the prenatal consultation process in Rio de Janeiro.

KEY WORKS: nursing consultation, prenatal, nursing, obstetric nursing 
RESUMEN: Consiste en un relato de experiencia sobre un proyecto de investigación que empezó en agosto de 1997 e involucra profesores del Departamento Materno-infantil de la Facultad de Enfermería de la UERJ y profesionales del Centro Municipal de Salud de Rio de Janeiro. Tiene como objetivo describir el proceso de la inserción de las Consultas de Enfermería de Prenatal en los contenidos programáticos del curso, asi como su estructuración e implantación en el referido Centro Municipal de Salud. Ese trabajo propone una revisión en el modelo biomédico de las consultaciones para proporcionar rupturas de paradigmas y con eso auxiliar el aprendizaje y ampliar el acompañamiento prenatal en Rio de Janeiro.

PALABRAS CLAVE: consultas de enfermeria, prenatal, enfermeria obstetrica

\section{BIBLIOGRAFIA}

ÁVILA, Maria Betânia. PAISM - um programa de saúde para o bem estar de género. 2. ed. Recife: SOS CORPO, 1995.

BRASIL, Ministério da Saúde. Programa de Assistência Integral à Saúde da Mulher: Bases de Ação Programática . Brasilia, 1984.

BRASIL, Ministério da Saúde. Pré-natal de baixo risco - normas e manuais técnicos. Brasilia, 1986. COSTA, Ana Maria. O PAISM: uma história sem fim. 3. ed. Recife: SOS CORPO, 1995.

CRANCH, G.S. Funciones de enfermería/ obstetricia en los servicios maternoinfantilis . In: SEMINẢRIO SOBRE ASPECTOS DE ENFERMERIA - OBSTETRICIA EN SALUD MATERNOINFANTIL Y PLANIFICACIÓN DE LA FAMILIA, Washington, D.C., Organización Panamericana de la Salud,

1974. p.33-38. (Publicación científica-).

FALEIROS, Vicente de P. Saber profissional e poder institucional. 4. ed. São Paulo: Cortez, 1993.

FONTES, José Américo S. Assistência materno-infantil. Rio de Janeiro: Cultura médica, 1984.

ISRAEL, Giselle R. Mulher e Saúde: práticas educativas em 11 municípios. (Org.) Giselle R. Israel e Vitória R. o. Vellozo. Rio de Janeiro: IBAM/ENSUR/NEMPP, 1996.

MAMEDE, Marli V. e LESCURA, Yara. Educação em saúde: abordagem para o enfermeiro. São Paulo: Savier, 1990.

MARANHĀO, Amélia Maria S. A. et al. Atividades da enfermeira obstetra no ciclo gravídico-puerperal. São Paulo: E.P.U.,1990.

NOGUEIRA, Maria Inês. Assistência pré-natal - prática de saúde a serviço da vida.São Paulo: Hucitec, 1994.

PENNA, Lucia Helena G. Dez anos de PAISM no contexto do Sistema Único de Saúde - análise critica sobre o cuidado prestado nestes programas. Rio de Janeiro: Caderno de Pesquisa - cuidado é fundamental, EEAP/UNIRIO/NUPENF, Ano 2, nº 01,1998.

FIOCRUZIMS. O holocausto materno no Brasil. Rio de Janeiro: Súmula/Radis, 1998.

VASCONCELLOS, Edmard M. Educação popular nos serviços de saúde. Săo Paulo: Hucitec,1989.

ZIEGEL, E.; CRANLEY, M,S,E, Enfermagem Obstétrica. 8. ed. Rio de Janeiro: Guanabara Koogan, 1985. 696 p. 